\title{
Article \\ Impact Testing of 3D Re-Entrant Honeycomb Polyamide Structure Using Split Hopkinson Pressure Bar
}

\author{
Jiangping Chen ${ }^{1}$, Weijun Tao ${ }^{2, *}$ and Shumeng Pang ${ }^{3}$ \\ 1 School of Civil Engineering and Architecture, Guangzhou City Construction College, \\ Guangzhou 510925, China; chenjiangping@e.gzhu.edu.cn \\ 2 Key Laboratory of Earthquake Engineering and Control, Earthquake Engineering Research \& Test Center, \\ Guangzhou University, Guangzhou 510405, China \\ 3 School of Environment and Civil Engineering, Dongguan University of Technology, Dongguan 523808, China; \\ pangshumeng@e.gzhu.edu.cn \\ * Correspondence: twj2016@gzhu.edu.cn
}

Citation: Chen, J.; Tao, W.; Pang, S. Impact Testing of 3D Re-Entrant Honeycomb Polyamide Structure Using Split Hopkinson Pressure Bar. Appl. Sci. 2021, 11, 9882. https:// doi.org/10.3390/app11219882

Academic Editor: Ana M. Camacho

Received: 2 October 2021

Accepted: 19 October 2021

Published: 22 October 2021

Publisher's Note: MDPI stays neutral with regard to jurisdictional claims in published maps and institutional affiliations.

Copyright: (C) 2021 by the authors. Licensee MDPI, Basel, Switzerland. This article is an open access article distributed under the terms and conditions of the Creative Commons Attribution (CC BY) license (https:// creativecommons.org/licenses/by/ $4.0 /)$.

\begin{abstract}
In this study, a total of 30 3D re-entrant honeycomb specimens made of polyamide were fabricated with various configurations by using the additive manufacturing (AM) technique. Split Hopkinson Pressure Bar (SHPB) tests were conducted on the RH specimens at different impact velocities. The incident, reflected and transmitted waveforms can well explain the wave propagation and energy absorption characteristics of the specimens, which can help us to understand and analyse the process of impact loading. The stress-strain curves, energy absorption ability and failure modes of SHPB tests with different impact velocities and quasi-static compression tests were analysed and compared, and it was found that the flow stress and energy absorption ability of the specimens subjected to impact load were much improved. Among the tested specimens, specimen $\mathrm{C} 2$, with a smaller re-entrant angle $\theta$, displayed the best energy absorption ability, which was $1.701 \mathrm{~J} / \mathrm{cm}^{3}$ at the impact velocity of $22 \mathrm{~m} / \mathrm{s}$ and was 5.1 times that in the quasi-static test. Specimen C5 had the longest horizontal length of the diagonal bar $L_{0}$, and its energy absorption was $1.222 \mathrm{~J} / \mathrm{cm}^{3}$ at the impact velocity of $22 \mathrm{~m} / \mathrm{s}$ and was 15.7 times that in the quasi-static test, reflecting the superiority of a structurally stable specimen in energy absorption under impact loading. The test results can provide a reference for the optimization of the design of the same or similar structures.
\end{abstract}

Keywords: SHPB impact test; 3D re-entrant honeycomb; additive manufacturing; dynamic response; energy absorption

\section{Introduction}

Both auxetic materials and structures exhibit the characteristic of a negative Poisson's ratio, expanding in the lateral direction when stretched and contracting when compressed, which is contrary to the positive Poisson's ratio properties of general materials and structures and is also known as auxetic behaviour. The negative Poisson's ratio is a scale-independent property in the theory of elasticity, which means the auxetic behaviour can be obtained at different scale levels [1,2]. Due to the auxetic characteristic, it has some excellent properties, such as increased shear modulus [3,4], increased indentation resistance [3,5], high fracture toughness [6,7], high energy absorption [8,9] and unique acoustic absorption [10]. Therefore, it is widely used in aerospace, protection, biomedicine, manufacturing, sports, sensors and other fields [1].

Over the last three decades, many studies have been conducted on the theoretical, experimental and numerical simulation of negative Poisson's ratio materials and structures. Rad et al. [11] dealt with the calculation of the basic mechanical properties of RH structures by using the energy methods of solid mechanics along with numerical methods. Li et al. [12] theoretically analysed the three-dimensional (3D) RH auxetic structures based on both a small deflection model and large deflection model. Wang et al. $[13,14]$ established 
an analytical model of a 3D RH auxetic cellular structure based on the energy method to study the mechanical properties and introduced the interlocking assembly method into the fabrication of 3D periodic auxetic cellular structure. In the work of Dikshit et al. $[15,16]$, 3D-printed trapezoidal sandwich structures were designed to determine the quasi-static indentation response and failure mode, and 3D-printed corrugated sandwich structures were designed to monitor the crack initiation and crack propagation using the acoustic emission technique. Elipe et al. [17] present a comparative study of two-dimensional (2D) and 3D auxetic geometries by using CAD-CAE tools. However, there are still many problems to be further explored. In terms of application, although there are many potential applications, most of them remain in the theoretical and experimental stage. The immature research on the basic characteristics of 3D materials and structures such as dynamic mechanical behaviours, and the high manufacturing cost limit their practical application. The 3D re-entrant honeycomb $(\mathrm{RH})$ structure is a typical auxetic structure, as shown in Figure $1 \mathrm{~b}$. The deep study on its static and dynamic mechanical behaviours will help us to further explore other auxetic materials and structures. Although many related studies have been carried out on the RH structure, most of them are based on the 2D structure [18-21], and studies on the dynamic properties of the 3D RH structure are rare. The limited research on 3D RH dynamic experiments is mainly focused on sandwich plates with an auxetic core [22,23], while the research on the 3D RH structure itself is very limited [24].

On the other hand, with the rise of the additive manufacturing (AM) technique in recent years, it is possible to manufacture complex 3D structures. Among the materials used in AM, polymers [25,26] are by far the most utilized class of materials for AM [26]. Polyamide, as one of the dominant market shares of polymers, has high strength, high stiffness and excellent long-term performance [27]. It is worth noting that there is no previous study on the dynamic properties of a 3D RH structure made of polyamide in the literature. Therefore, it is necessary to study the dynamic properties of the newly developed 3D re-entrant structure made of polyamide under impact loading.

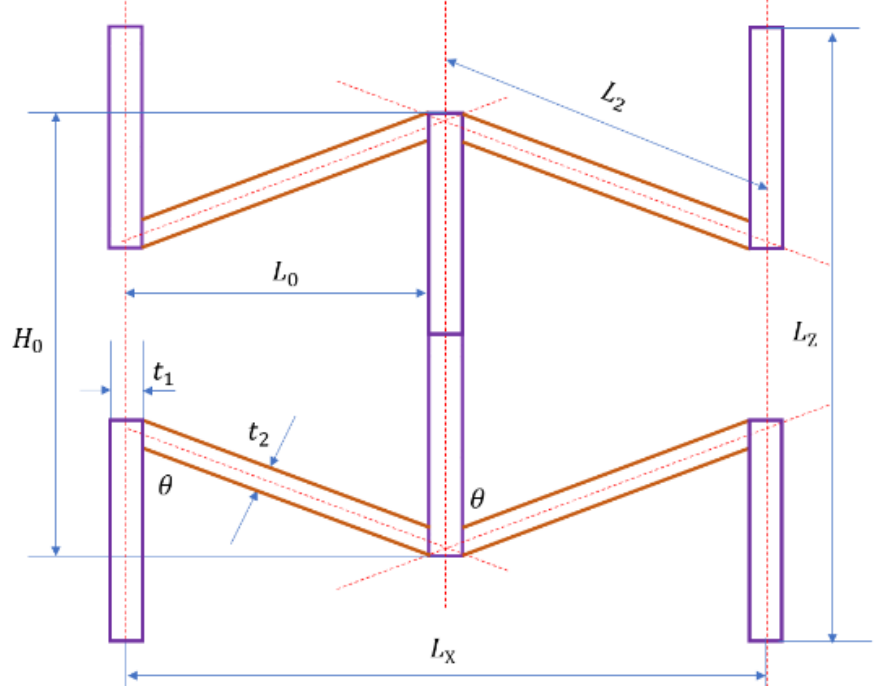

(a)

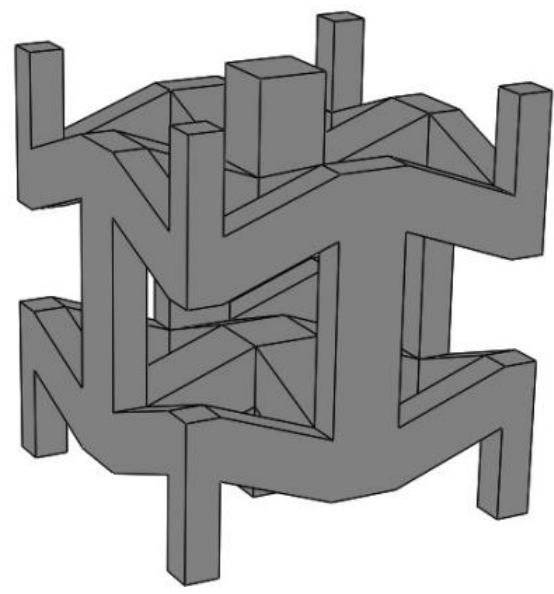

(b)

Figure 1. (a) Geometric parameters of unit cell [21]; (b) 3D unit cell [22].

In this study, 3D RH auxetic structures made of polyamide were further studied for their dynamic impact properties on the basis of the previous study on their quasi-static mechanical properties [28]. Firstly, 3D RH polyamide structure specimens were printed by AM technology. Then, Split Hopkinson Pressure Bar (SHPB) technology was used to conduct impact tests on specimens with various geometric configurations. The dynamic test results were compared with the quasi-static test results. This study aimed to reveal 
the dynamic mechanical properties of the 3D RH specimens, and provide basic data and technical support for the study of this kind of auxetic structure.

\section{Related Theories on the SHPB Test}

SHPB technology is widely used to carry out impact tests on specimens to obtain the dynamic properties of materials. SHPB is usually made of high-strength steel; however, the specimens in this study are of a 3D RH polyamide structure, and the mechanical impedance and strength of the specimens are much lower than that of steel bar. If the SHPB steel bar with high impedance acts on the specimen with low impedance, the incident waveform and reflected waveform will be very close, and the transmissive wave signal will be too weak to be detected accurately. Therefore, an SHPB bar whose impedance is close to the specimen should be used [29-31]. In this study, the viscoelastic polymethyl methacrylate (PMMA) bar was used, and the mechanical impedance of the PMMA bar was relatively matching with the specimen. It should be noted that the wave attenuation caused by material damping and the wave dispersion effect caused by radial inertia should be considered on the wave propagation of the viscoelastic bar. Bacon [29] developed an experimental method to evaluate the propagation coefficient. Zhao and Gary [32] presented an analytical solution of the longitudinal wave propagation in a viscoelastic cylindrical bar. Wang et al. [31,33] developed a method based on the Zhu-Wang-Tang (ZWT) viscoelastic constitutive equation and the characteristic theory of wave propagation. According to some studies [34], if the minimum wavelength of the impact wave is significantly greater than the lateral dimensions of the bar, the dispersion effect caused by radial inertia can be neglected.

For wave attenuation caused by material damping, the waveform at different locations varies with distance within a limited propagation distance (up to $3 \mathrm{~m}$ in bar length). Therefore, the waveform measured by the test at the position of strain gauge on the SHPB bar cannot directly replace the waveform at the interface between the bar and the specimen. In this study, an improved Lagrange analysis method [35], which was verified in an earlier study, was used to process the strain data measured on the SHPB bar, and then the incident strain wave $\varepsilon_{I}\left(X_{1}, t\right)$ and transmission strain wave $\varepsilon_{T}\left(X_{2}, t\right)$ at the two interface sites could be obtained, respectively. The wave dispersion effect caused by radial inertia was neglected, and the ZWT viscoelastic constitutive equation and characteristic method were used to solve the strain, stress and velocity of all positions on the PMMA bar. These data were compared with the data obtained by the improved Lagrange analysis method to verify the validity of dynamic data processing on the PMMA bar. It is worth noting that this paper mainly studies the impact behaviour of the 3D RH structure, rather than the dynamic testing process and data processing of the PMMA bar. The corresponding content will be detailed in other papers published by the authors.

Based on the one-dimensional stress wave hypothesis, the stress and strain of the SHPB impact specimen can be calculated by using the one-dimensional stress wave theory, which can be expressed as:

$$
\begin{gathered}
\sigma_{s}(t)=\frac{E A}{A_{s}}\left[\varepsilon_{I}\left(X_{1}, t\right)+\varepsilon_{R}\left(X_{1}, t\right)\right]=\frac{E A}{A_{s}} \varepsilon_{T}\left(X_{2}, t\right), \\
\varepsilon_{s}(t)=\frac{2 C_{0}}{l_{s}} \int_{0}^{t} \varepsilon_{R}\left(X_{1}, t\right) d t=\frac{2 C_{0}}{l_{s}} \int_{0}^{t}\left[\varepsilon_{T}\left(X_{2}, t\right)-\varepsilon_{I}\left(X_{1}, t\right)\right] d t
\end{gathered}
$$

where $\sigma_{s}(t)$ and $\varepsilon_{s}(t)$ are the average stress and strain of the specimen, respectively. $A_{s}, l_{s}$ represent the cross-section area and length of the specimen, respectively. $E, A, C_{0}$ represent the Young's modulus, the cross-section area and the longitudinal elastic wave velocity of bar, respectively. 


\section{Materials and Methods}

\subsection{Specimen and Material}

The 3D RH polyamide specimens still used the same structural form as the quasistatic test. The unit cell is shown in Figure 1, and the sample photographs are shown in Figure 2. The specimens were made of polyamide (PA2200) material which was supplied by the supplier EOS, Germany, and were fabricated by using additive manufacturing. The material properties of polyamide are listed in Table 1.
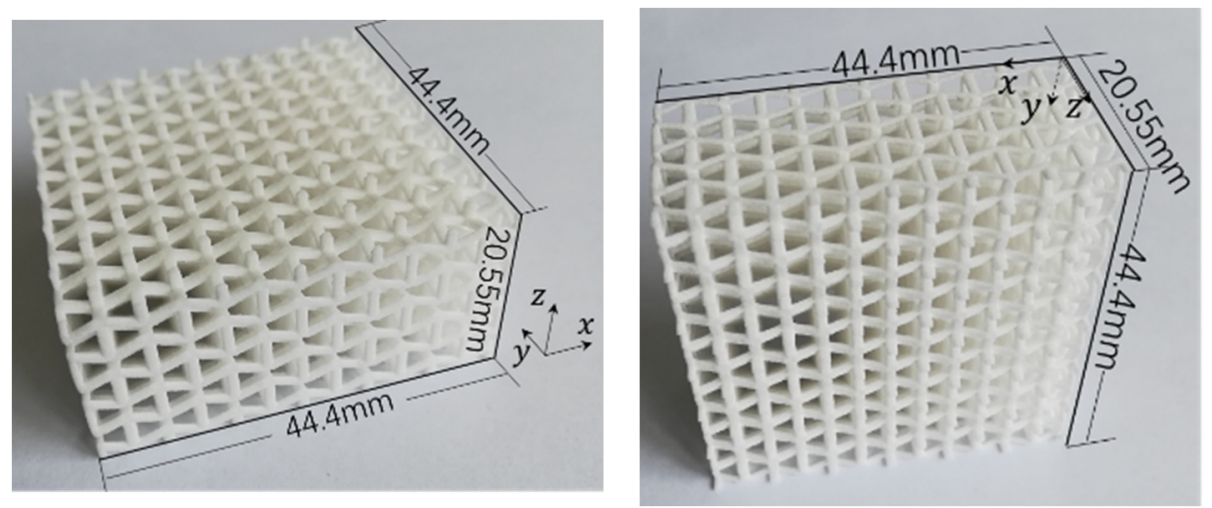

Figure 2. Photograph of sample specimen (C4).

Table 1. Material properties of polyamide [27].

\begin{tabular}{ccccc}
\hline Material & Young's Modulus E $\mathbf{( M P a )}$ & Tensile Strength $\boldsymbol{\sigma}_{\mathbf{B}} \mathbf{( M P a )}$ & Elongation $\varepsilon_{\mathbf{B}}(\mathbf{\%})$ & Density $^{\left(\mathrm{g} / \mathrm{cm}^{\mathbf{3}}\right)}$ \\
\hline PA 2200 & $1500 \sim 1650$ & 48 & 18 & 0.93 \\
\hline
\end{tabular}

A total of 30 polyamide specimens with five configurations (i.e., C1-C5) were designed, with 6 of the same specimens per configuration. The parameters are given in Table 2, and the design variables are indicated in bold. It is worth noting that, due to the size limitation of the SHPB test equipment, the unit size of the specimen in the SHPB test was set to one third of that of the quasi-static test specimen, that is, to reduce the unit size in the same proportion in three directions. Each specimen had the array of $N_{\mathrm{X}} \times N_{\mathrm{y}} \times N_{\mathrm{z}}$ unit cells. Four main parameters, displayed in grey, were taken into consideration, i.e., the length of the vertical strut $\left(H_{0}\right)$, the horizontal length of the oblique strut $\left(L_{0}\right)$, the re-entrant angle between the vertical strut and the oblique strut $(\theta)$ and the thickness of the oblique strut cross section $\left(t_{2}\right)$, as shown in Figure $1 \mathrm{a} . \mathrm{C} 1$ and $\mathrm{C} 2$ were designed to investigate the effect of the re-entrant angle $\theta$. C1, C4 and C5 were designed to investigate the effect of $L_{0}$. C3 was designed to investigate the effects of both $t_{2}$ and $\theta$.

Table 2. Design parameters for each specimen (C1-C5).

\begin{tabular}{ccccccccccc}
\hline Specimen & $\boldsymbol{t}_{\mathbf{1}}(\mathbf{m m})$ & $\boldsymbol{t}_{\mathbf{2}}(\mathbf{m m})$ & $\boldsymbol{\theta}\left({ }^{\circ}\right)$ & $\boldsymbol{L}_{\mathbf{0}}(\mathbf{m m})$ & $\boldsymbol{H}_{\mathbf{0}}(\mathbf{m m})$ & $\boldsymbol{L}_{\boldsymbol{x}}(\mathbf{m m})$ & $L_{\boldsymbol{z}}(\mathbf{m m})$ & $N_{\boldsymbol{x}}, N_{\boldsymbol{y}}$ & $\boldsymbol{N}_{\boldsymbol{z}}$ \\
\hline $\mathrm{C} 1$ & 1.2 & 1.0 & $\mathbf{7 0}$ & 2.6 & 5.4 & 6.4 & 7.22 & 7 & 3 \\
\hline $\mathrm{C} 2$ & 1.2 & 1.0 & $\mathbf{6 0}$ & 2.6 & 5.4 & 6.4 & 6.18 & 7 & 3 \\
\hline $\mathrm{C} 3$ & 1.2 & $\mathbf{0 . 5}$ & $\mathbf{5 0}$ & 2.6 & 6.0 & 6.4 & 7.34 & 7 & 3 \\
\hline $\mathrm{C} 4$ & 1.2 & 1.0 & 70 & $\mathbf{3 . 1}$ & 5.4 & 7.4 & 6.85 & 6 & 3 \\
\hline $\mathrm{C} 5$ & 1.2 & 1.0 & 70 & $\mathbf{3 . 6}$ & 5.4 & 8.4 & 6.49 & 5 & 3 \\
\hline
\end{tabular}

Note: Please refer to Figure 1 for the notations. 


\subsection{SHPB Setup}

SHPB tests were carried out on the SHPB testing system of Guangzhou University. The experimental setup is shown in Figure 3. It consists of a power system, striking bar, incident bar, transmission bar, absorber and measurement and recording system. Both the incident bar and transmission bar are viscoelastic PMMA bars with a length of $2 \mathrm{~m}$ and a diameter of $40 \mathrm{~mm}$. The striking bar is a viscoelastic PMMA bar with a length of $0.4 \mathrm{~m}$ and a diameter of $40 \mathrm{~mm}$. The ends of the above three bars are flat. Three strain gauges were placed on the incident bar and the transmission bar, respectively, with a spacing of $10 \mathrm{~cm}$ of the strain gauge on the incident bar and $20 \mathrm{~cm}$ of the strain gauge on the transmission bar. The specimen was placed between the incident bar and the transmission bar, as shown in Figure 3. A pulse-shaper was placed on the impact face of the incident bar to reduce the wave oscillation and dispersion effect in the incident bar.

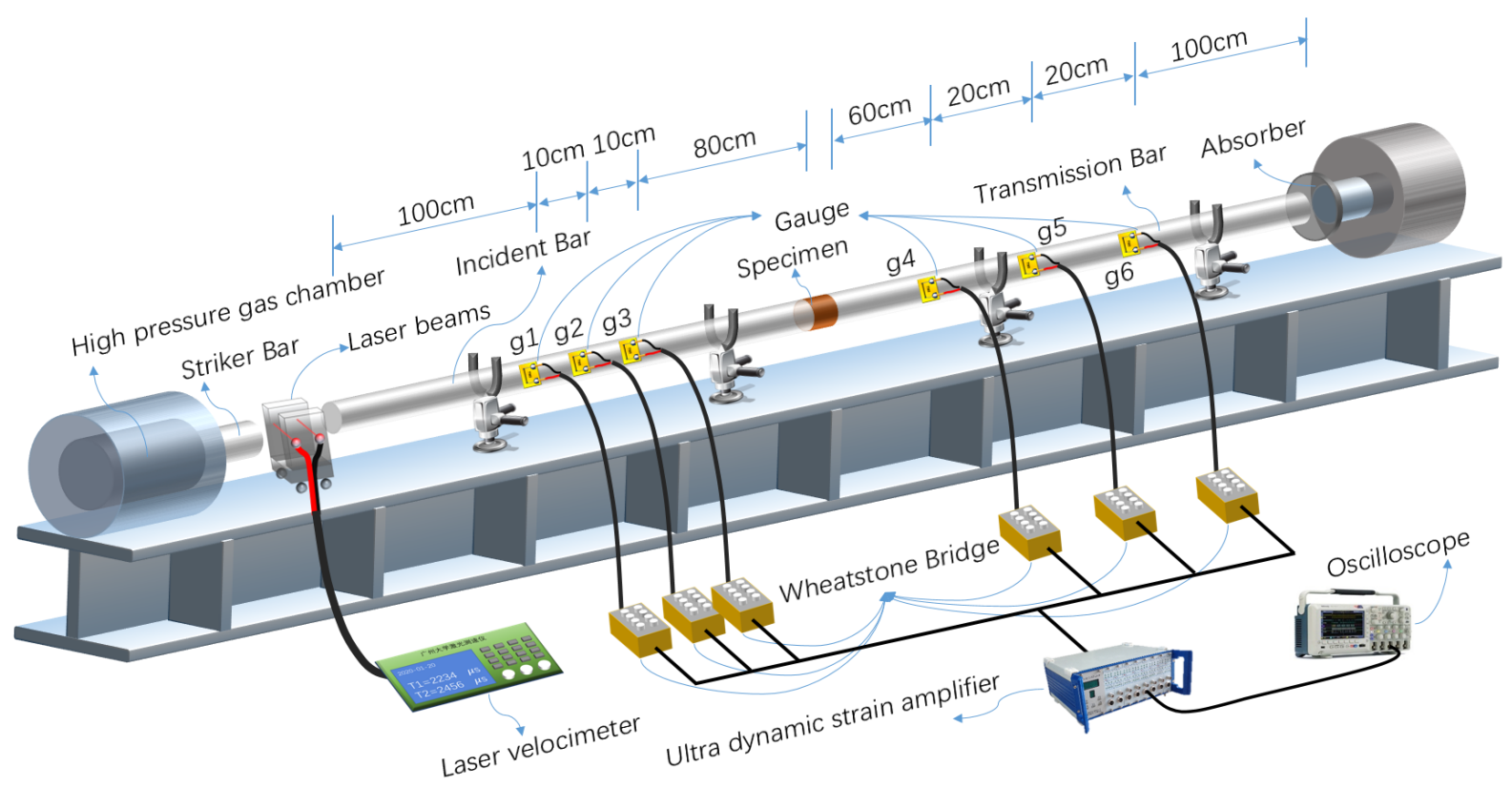

Figure 3. SHPB experimental setup.

\subsection{SHPB Tests}

Series of preparations are needed before the SHPB tests of polyamide specimens can be formally started. Firstly, static calibration was carried out for the circuit affixed with strain gauges on the PMMA bar to verify the correctness of the circuit system. Secondly, the calibration of the laser velocimeter was carried out, combining the laser distance test with the interval time measured by the laser velocimeter to calibrate the bullet impact velocity. Thirdly, the relationship between the strain caused by bullet impacting and the voltage displayed by the oscilloscope was established, and dynamic calibration of the strain gauge was carried out to correct the relationship between bullet impact velocity and voltage. Finally, because the PMMA bar is a kind of viscoelastic bar, wave attenuation caused by material damping will occur when the wave is propagating; therefore, the dynamic mechanical properties of the PMMA bar itself need to be tested.

In the dynamic tests for the PMMA bar, the above SHPB experimental setup was adopted, but the incident bar was directly connected with the transmission bar without a specimen between them. The initial velocity of the striking bar was set to $22 \mathrm{~m} / \mathrm{s}$. The strain time-history curve was calculated by the data acquisition system. Then, the improved Lagrange analysis method, which was verified in [35], was used to process the strain time-history data and obtained the stress time-history and velocity time-history data. Next, the ZWT viscoelastic constitutive equation and characteristic method were adopted 
to verify the effectiveness of these data. The results were found to be in good agreement, reflecting the effectiveness of dynamic data processing on the PMMA bar.

SHPB tests for the specimens were conducted along the $\mathrm{Z}$ direction. The working conditions were distinguished by the impact velocity of the striker bar, which were $14 \mathrm{~m} / \mathrm{s}$, $18 \mathrm{~m} / \mathrm{s}$ and $22 \mathrm{~m} / \mathrm{s}$, respectively. Two specimens with the same configuration were tested for each working condition, and the calculated results were averaged. The longitudinal strain was measured by the six strain gauges located on the incident bar and transmission bar. The gauges were connected to a dynamic bridge amplifier. The amplified strain was recorded by a digital oscilloscope. Then, the signal from the oscilloscope was transferred to a computer for data processing and the strain time-history $\varepsilon(t)$ at both ends of the specimen could be obtained.

\section{Results and Discussion}

\subsection{Waveform Analysis}

Figure 4 shows the incident, reflected and transmitted waveforms measured by oscilloscope in the specimen $\mathrm{C} 2$ test at the working condition of $\mathrm{v}=18 \mathrm{~m} / \mathrm{s}$. Three incident and reflected waveforms can be measured from the strain gauges $X_{1}, X_{2}$ and $X_{3}$ on the incident bar. Three transmitted waveforms can be measured from the strain gauges $X_{4}, X_{5}$ and $X_{6}$ on the transmission bar. It can be found that the trend, the pulse width and the amplitude of these waveforms all follow similar rules and have good consistency, which can reflect the wave propagation process in the viscoelastic PMMA bar and the energy absorption characteristics of specimens.

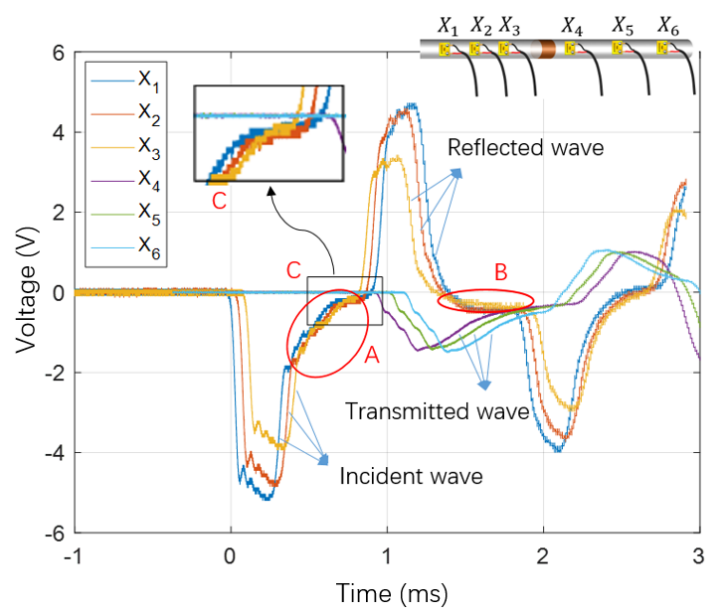

Figure 4. The voltage signal measured by $\mathrm{C} 2(\mathrm{v}=18 \mathrm{~m} / \mathrm{s})$.

Based on the tests on the PMMA bar, the material constants of the PMMA bar $\left(E_{0}=3.19 \mathrm{Gpa}, \rho_{0}=1.176 \mathrm{~kg} / \mathrm{m}^{3}\right.$ ) can be obtained, and then the wave propagation velocity $\left(C_{0}=\sqrt{E_{0} / \rho_{0}}=1647 \mathrm{~m} / \mathrm{s}\right)$ can be calculated as well. As the length of the striker bar is $40 \mathrm{~cm}$, the pulse width $\left(\mathrm{t}=2 L_{0} / C_{0}=0.486 \mathrm{~ms}\right)$ can be obtained. It can be observed from Figure 4 that the pulse width of the incident wave is basically consistent with the calculated pulse width in an elastic state, which verifies the validity of the test.

The incident bar receives the compression wave caused by the incident pulse under the impact of the striker bar. The compression wave reaches the strain gauges $X_{1}, X_{2}$ and $X_{3}$ in turn. It can be seen from Figure 4 that the incident wave gradually attenuates in the PMMA bar, i.e., the wave amplitude tends to decrease along the direction of wave propagation. It also can be seen that the wave attenuation is not proportional to the propagation distance. In addition, at the end of the incident pulse, the compression wave does not immediately unload to 0 , but presents a "creep" characteristic with time growth, that is, so-called compression strain delay, as shown from ellipse A in Figure 4. It can be interpreted that the viscosity of the PMMA bar is the reason for the "creep" phenomenon without the influence 
of other external factors. Subsequently, the specimen deforms at high speed under the action of the incident wave, and propagates the reflected stretch wave to the incident bar and the transmitted compression wave to the transmission bar simultaneously.

The reflected wave reaches back to the strain gauges $X_{3}, X_{2}$ and $X_{1}$ in turn. As can be seen from the partial enlarged subgraph, namely rectangle box $C$, when the reflected wave reaches the strain gauge for the first time, the compression "creep" caused by the incident wave is still incomplete, resulting in the superposition of the head of the reflected wave and the tail of the incident wave. From the time period between the arrival of the incident wave and the arrival of reflected wave, it can be found that it spends the longest time when the reflected wave reaches the position of strain gauge $X_{1}$. At this time, the compression deformation caused by the incident wave has basically completed; that is, the "creep" effect is minimal. However, the "creep" at the position of strain gauge $X_{3}$ is maximal, and the deformation hysteresis is not completed before it enters the stretch wave. After one pulse width, the tail of the reflected wave passes through the strain gauge. It can be observed from the B ellipse in Figure 4 that the reflected wave does not immediately unload to 0 , but directly changes from the stretch wave to the compression wave. This phenomenon indicates that the incident bar is under pressure after the tail of the first reflected wave. It can be interpreted that the high-speed deformation of the specimen causes it to compress and absorb a lot of impact energy, including elastic and plastic potential energy. Then, the elastic potential energy is released and acts on the incident bar to place it in a compression state.

The transmission bar receives the transmitted compression wave. The transmitted wave reaches the strain gauges $X_{4}, X_{5}$ and $X_{6}$ in turn. As these three strain gauges are spaced $20 \mathrm{~cm}$ apart, it was shown in Figure 4 that the transmitted wave spacing was about twice that of the incident wave or the reflected wave. In order to more clearly express the transmitted wave, the transmitted waveforms are taken out for analysis separately, as shown in Figure 5. It can be seen the three transmitted waveforms all have the super-long pulse width which is much longer than the loading pulse width, that is, the so-called extended pulse width. The extended part is shown in the rectangle box D in Figure 5, with a total pulse width of about $1.4 \mathrm{~ms}$. The reasons for the occurrence of the super-long pulse width are as follows: on the one hand, the compression delay phenomenon appears in the rising process of the transmitted pulse, because after the incident wave is transmitted to the specimen interface, it passes through the specimen with relatively small impedance, and its propagation velocity slows down, leading to the increase in the pulse width of transmitted wave when it is transmitted to the transmission bar. On the other hand, when the specimen conducts the transmitted wave, it releases the absorbed elastic potential energy to the transmission bar, which causes the secondary compression of the transmission bar, resulting in the extension of the pulse width and compression "creep". After that, the transmitted wave reaches the end of the transmission bar and the sparse wave is reflected back, which passes through the strain gauges $X_{6}, X_{5}$ and $X_{4}$ in turn. As shown from the partial enlarged subgraph, namely rectangle box $E$, when the reflected sparse wave reaches the position of strain gauges for the first time, the compression "creep" caused by the transmitted wave is not completed, resulting in the superposition of the head of the sparse wave and the tail of the transmitted wave. It also can be seen that the transmitted wave does not immediately unload to 0 , but directly changes from the compression wave to the stretch wave. From the time period between the arrival of transmitted wave and the arrival of the sparse wave, it can be found that the pulse width at $X_{4}$ is the largest and the pulse width at $\mathrm{X}_{6}$ is the smallest. 


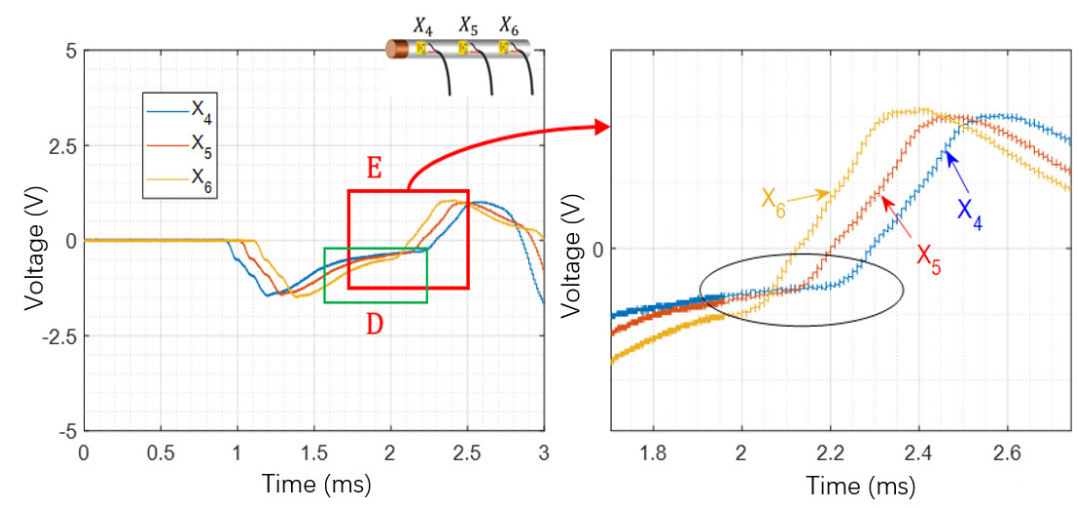

Figure 5. Comparison of three transmitted waveforms.

\subsection{Stress-Strain Curves}

The strain time-history curve at the position of the strain gauge of the PMMA bar was obtained from the voltage signal using the data acquisition system. The improved Lagrange analysis method [35] was used to process the strain data, and then the incident strain wave $\varepsilon_{I}\left(X_{1}, t\right)$ at the interface $X_{1}$ between the incident bar and the specimen, and the transmission strain wave $\varepsilon_{T}\left(X_{2}, t\right)$ at the interface $X_{2}$ between the transmission bar and the specimen, can be obtained. The stress and strain time-history curves of the tested specimen can be calculated by the traditional SHPB stress wave Equations (1) and (2).

Figure 6 shows the stress-strain curves of each specimen at impact velocities of approximately 14, 18 and $20 \mathrm{~m} / \mathrm{s}$. Generally speaking, the stress of each specimen under impact loading includes three obvious stages, namely elastic growth, plastic growth and decline. Moreover, each specimen completes elastic growth and enters plastic growth within a very short time after loading. Among them, specimen $C 2$ is the most impressive due to its appropriate re-entrant angle $\theta$ and maximum density (please refer to [28]). Its peak stress is the largest in all working conditions, and the peak stress increases with the increase in impact velocity. Due to the stable structural configuration, namely the longest horizontal length of the oblique strut $\left(L_{0}\right)$, specimen $C 5$ exhibits obvious and long plateau stress. Because the thickness of the oblique strut cross section $\left(t_{2}\right)$ of specimen $C 3$ is reduced to half of that of other specimens, the plastic stress increases gently and the peak stress is relatively low. The stress of C4 was unstable at different impact velocities. The peak stress of specimen $C 1$ is relatively low, and the peak stress increases with the increase in impact velocity.

Comparing the stress-strain curves of specimens $\mathrm{C} 1$ to $\mathrm{C} 2$, it is found that when the re-entrant angle $(\theta)$ changes from $70^{\circ}$ to $60^{\circ}$, it has a significant influence on the dynamic properties of the 3D RH structure under impact loading, which shows that the smaller re-entrant angle $\theta$ lead to a larger Young's modulus (the slope at the elastic growth stage) and larger peak stress, indicating the importance of an appropriate $\theta$ to the structure performance under impact loading. Compared with $\mathrm{C} 1, \mathrm{C} 4$ and $\mathrm{C} 5$, whose horizontal length of the oblique strut $\left(L_{0}\right)$ are $2.6 \mathrm{~mm}, 3.1 \mathrm{~mm}$ and $3.6 \mathrm{~mm}$, respectively, it can be found from Figure 6 that with the increase in $L_{0}$, except for $C 4$, which is unstable at v0 = $18 \mathrm{~m} / \mathrm{s}$, the peak stress increases and the plateau stress tends to be obvious. It is worth noting that C5 performs particularly well, although the density (please refer to [28]) of C5 is the smallest among all the specimens with different configurations; its stable structure causes it to perform well in both stress growth and energy absorption. Compared with C1, C2 and $\mathrm{C} 3$, whose re-entrant angle $(\theta)$ changes from $70^{\circ}$ to $60^{\circ}$ and then to $50^{\circ}$, and the fact that the thickness of the oblique strut cross section $\left(t_{2}\right)$ of specimen $\mathrm{C} 3$ is reduced to half of that of other specimens, it shows that due to the half-reduced $t_{2}$, the strength of specimen C3 is greatly reduced and C3 is vulnerable to shear failure, which indicates that the effect of decreasing $t_{2}$ is greater than that of decreasing $\theta$. 


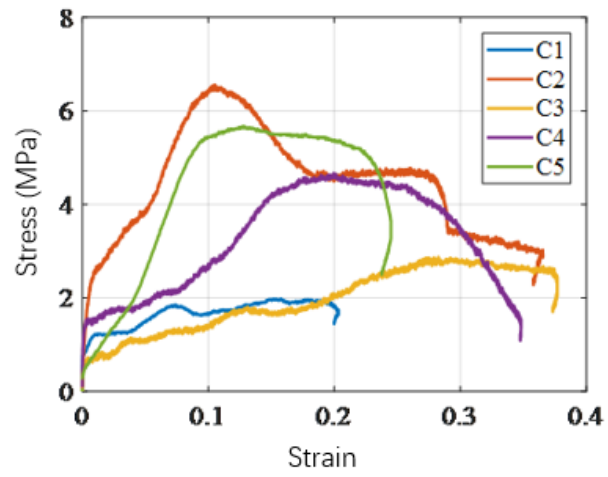

(a)

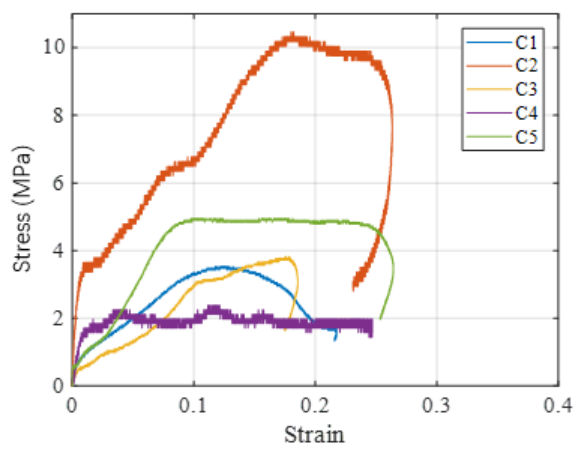

(b)

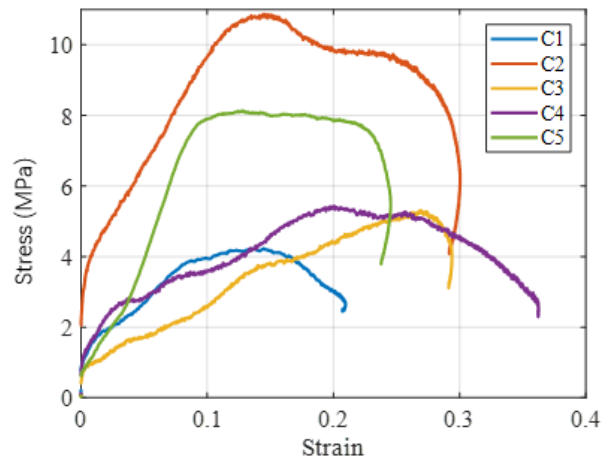

(c)

Figure 6. Stress-strain curves of the specimens under different working conditions. (a) v0 = $14 \mathrm{~m} / \mathrm{s}$, (b) $\mathrm{v} 0=18 \mathrm{~m} / \mathrm{s},(\mathbf{c}) \mathrm{v} 0=22 \mathrm{~m} / \mathrm{s}$.

In order to compare the mechanical properties of specimens under quasi-static and different impact velocities, Figure 7 compares the stress-strain curves of representative specimens (C2, C3 and C5) under different working conditions. The results are as follows: (1) With the increase in impact velocity, the peak stress of the specimen increases and shows the strain rate sensitivity, and the yield stress increases gradually with the increase in strain rate; (2) Compared with the quasi-static compression, the yield stress and peak stress under impact loading are greatly increased, reflecting the strain-hardening phenomenon under a high strain rate; (3) Different from the quasi-static compression test, there is an obvious decline stage in the stress-strain curves of the specimens under impact loading, indicating that the specimens experience overall failure after energy absorption by impact, while the quasi-static specimens are in a continuous compaction state.

\subsection{Energy Absorption}

Figure 8 shows the energy absorption curves of different specimens at different impact velocities. Energy absorption is defined as the area enclosed by the stress-strain curves at a certain strain, namely, deformation energy per unit volume. In general, when the strain value is the same, C2 has the best energy absorption capacity, C5 comes second, and $\mathrm{C} 3$ has the worst. Therefore, when the structure has no obvious defect, the smaller the re-entrant angle $(\theta)$ or the more stable the structure, the stronger the energy absorption capacity; when the structure has obvious defects, the energy absorption capacity is poor. 


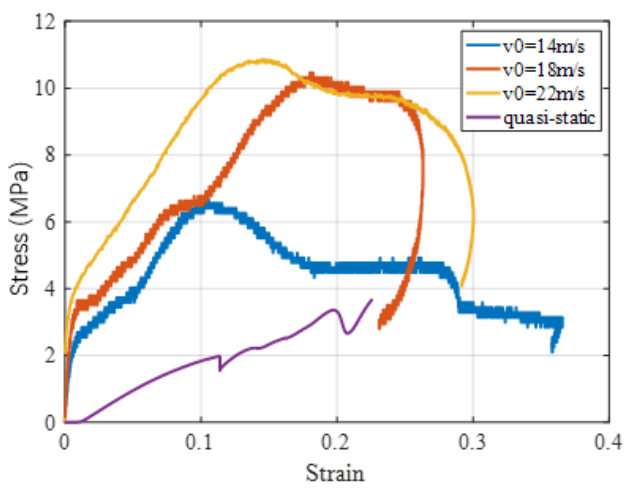

(a)

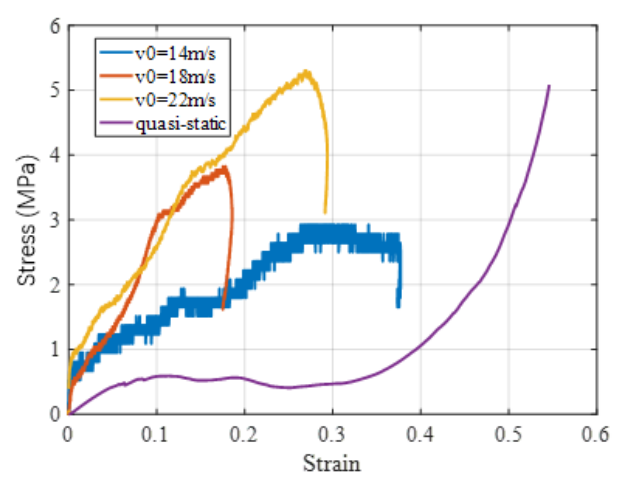

(b)

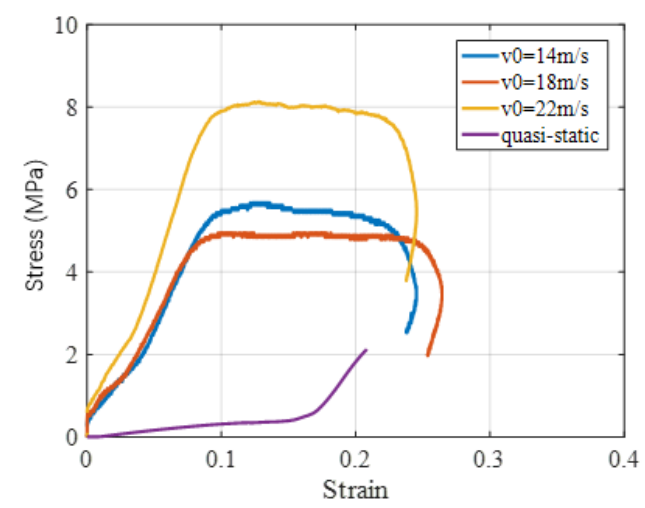

(c)

Figure 7. Comparison of stress-strain curves of specimens under different working conditions. (a) $\mathrm{C} 2,(\mathbf{b}) \mathrm{C} 3,(\mathbf{c}) \mathrm{C} 5$.

Figure 9 compares the energy absorption of specimens C2, C3 and C5 under different impact velocities and quasi-static tests. On the whole, the energy absorption capacity of specimens under impact loading is significantly enhanced compared with that of quasistatic specimens; the larger the impact velocity is, the more energy is absorbed by the specimen. Table 3 compares the energy absorption capacity of the specimens under SHPB tests and quasi-static compression tests at the same strain of 0.2. It can be found that the energy absorption capacity of the SHPB specimen is several times higher than that of the quasi-static specimen. Among them, the most stable structural impact specimen, C5, is particularly outstanding, with the highest energy absorption ratio of 15.7 times that of the corresponding quasi-static specimen. Specimen C2 has the highest density, and its energy absorption capacity is the highest in both quasi-static and impact tests, which is $1.701 \mathrm{~J} / \mathrm{cm}^{3}$ at the impact velocity of $22 \mathrm{~m} / \mathrm{s}$ and is 5.1 times that in the quasi-static test. Specimen C5 has the lowest density, and its energy absorption capacity is the lowest in the quasi-static test; however, it has the second highest energy absorption capacity in the impact test, of $1.222 \mathrm{~J} / \mathrm{cm}^{3}$ at the impact velocity of $22 \mathrm{~m} / \mathrm{s}$, indicating the advantages of a stable structure in energy absorption under impact loading. 


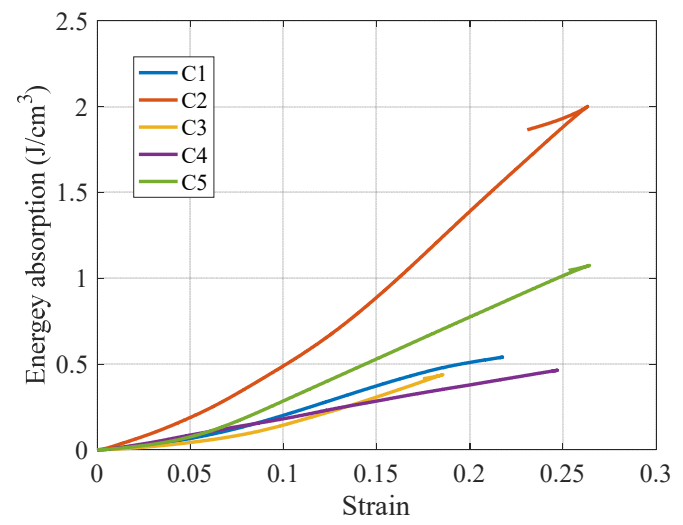

(a)

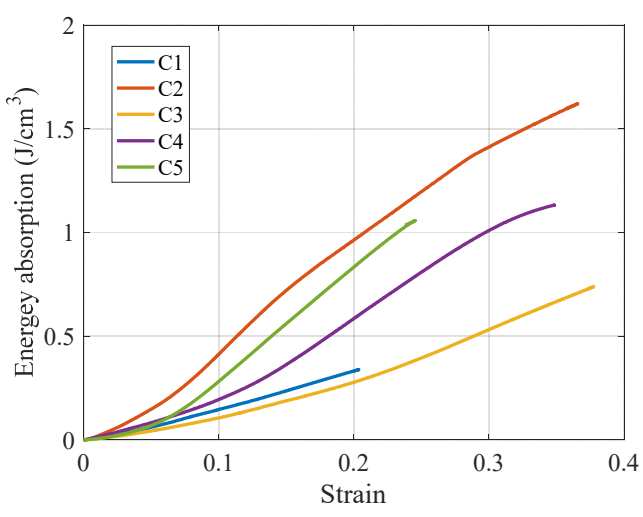

(b)

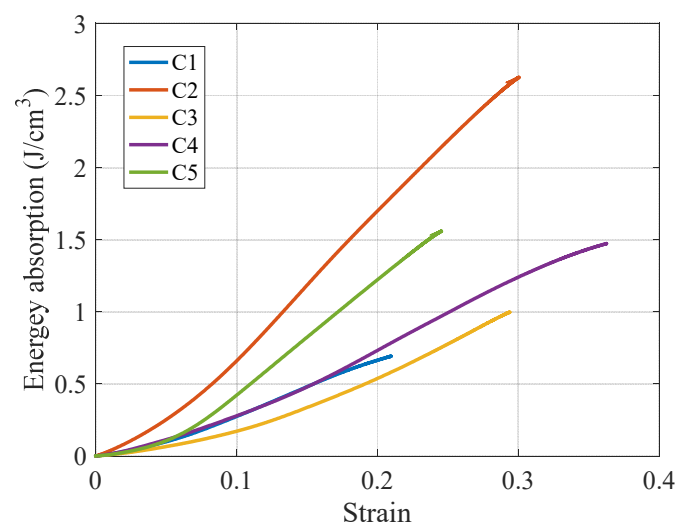

(c)

Figure 8. Energy absorption curves of different specimens at different impact velocities. (a) v0 = $14 \mathrm{~m} / \mathrm{s},(\mathbf{b}) \mathrm{v} 0=18 \mathrm{~m} / \mathrm{s}$, (c) $\mathrm{v} 0=22 \mathrm{~m} / \mathrm{s}$.

Table 3. Comparison of the energy absorption $\left(\mathrm{J} / \mathrm{cm}^{3}\right)$ of the specimens under SHPB tests and quasi-static compression tests.

\begin{tabular}{cccccc}
\hline $\mathbf{V}$ & $\mathbf{C 1}$ & $\mathbf{C 2}$ & $\mathbf{C 3}$ & $\mathbf{C 4}$ & $\mathbf{C 5}$ \\
\hline quasi-static & 0.109 & 0.333 & 0.092 & 0.145 & 0.078 \\
\hline $14 \mathrm{~m} / \mathrm{s}$ & $0.333 / 3.1$ & $0.934 / 2.8$ & $0.278 / 3.0$ & $0.585 / 4.0$ & $0.834 / 10.7$ \\
\hline $18 \mathrm{~m} / \mathrm{s}$ & $0.509 / 4.7$ & $1.389 / 4.2$ & $0.438 / 4.8$ & $0.378 / 2.6$ & $0.773 / 9.9$ \\
\hline $22 \mathrm{~m} / \mathrm{s}$ & $0.664 / 6.1$ & $1.701 / 5.1$ & $0.537 / 5.8$ & $0.733 / 5.1$ & $1.222 / 15.7$
\end{tabular}

Note: Under dynamic loading, the first value is the absorbed energy value, and the second value is the ratio of the absorbed energy of the impact specimen to that of the corresponding quasi-static specimen. 


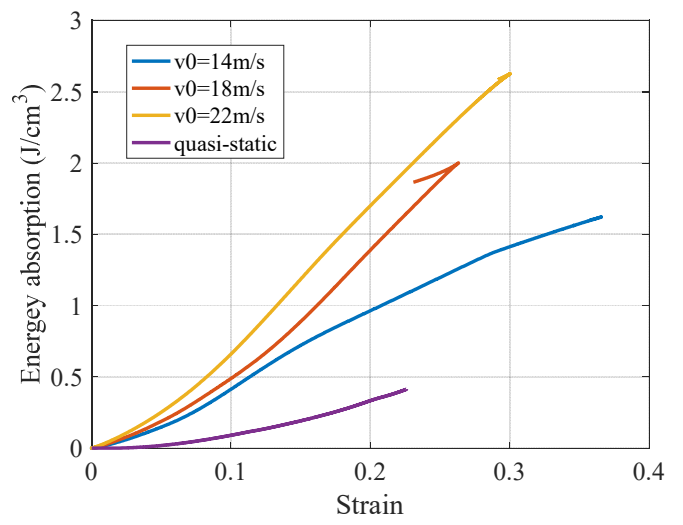

(a)

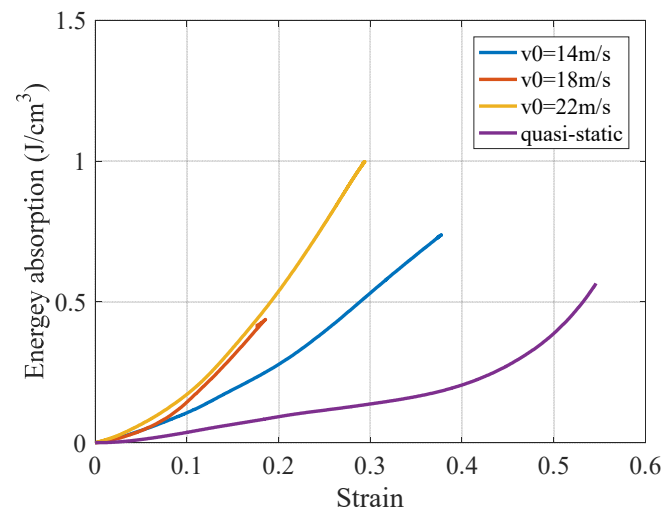

(b)

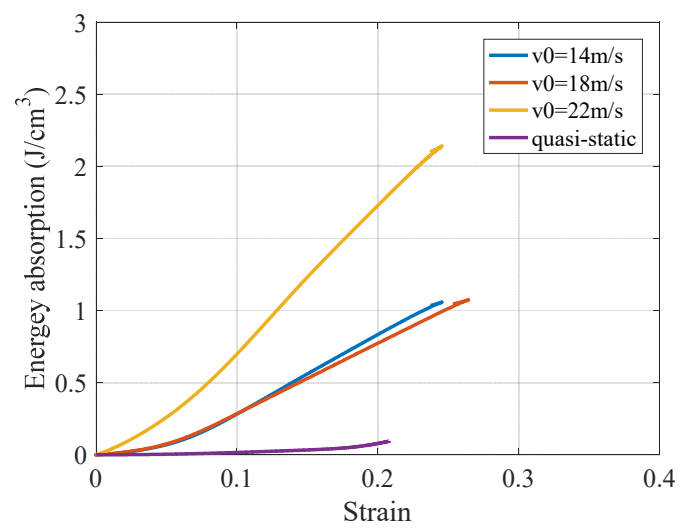

(c)

Figure 9. Comparison of the energy absorption of specimens under different impact velocities and quasi-static tests. (a) C2, (b) C3, (c) C5.

\subsection{Failure Mode}

The specimens exhibit a variety of dynamic failure modes under impact loading, including (1) local large plastic deformation; (2) dynamic brittleness caused by the strain rate effect; (3) adiabatic shear failure caused by the temperature effect; and (4) caving failure caused by stress wave interaction. The failure patterns of the impacted surface and the side of the impact specimen are shown in Figure 10a,b. It is easy to observe the local dent caused by the impact of the compression bar and the caving content caused by the structural fracture due to the tensile stress wave. In addition, with the increase in strain rate, the yield stress and flow stress increase correspondingly, which reduces the plastic deformation zone and leads to dynamic brittleness. Material deformation changes from isothermal deformation to adiabatic deformation, which leads to a temperature rise and the adiabatic shear failure of the specimen.

In contrast, the failure patterns of the compressed surface and the side of the quasistatic compression specimen are shown in Figure 10c,d. Due to the sufficient deformation time and insignificant temperature effect, the quasi-static compression specimen does not demonstrate caving failure, and its deformation is moderate and relatively uniform. 


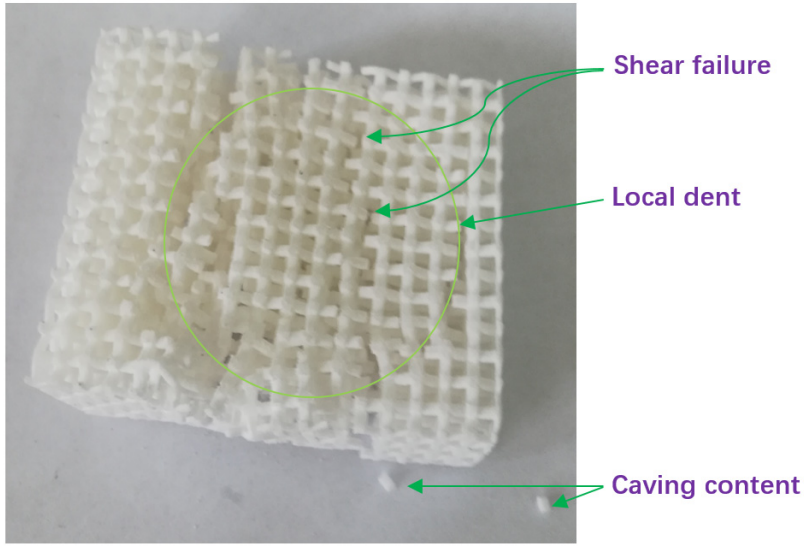

(a)

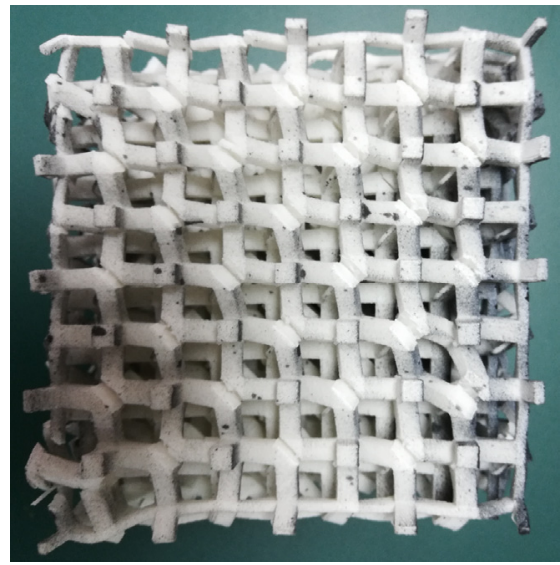

(c)

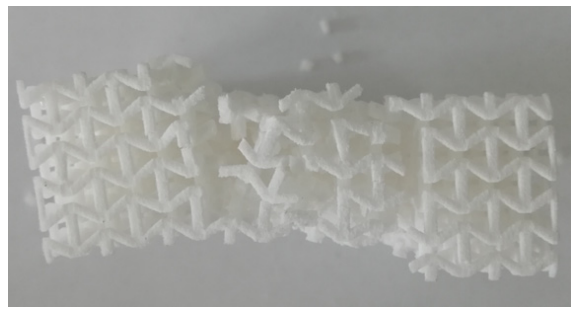

(b)

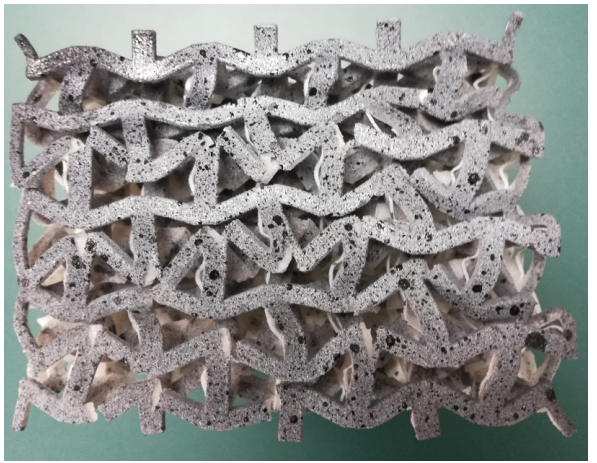

(d)

Figure 10. Failure patterns of specimens. (a) Impacted surface of impact specimen. (b) Side surface of impact specimen. (c) Compressed surface of quasi-static specimen. (d) Side of quasi-static specimen.

\section{Conclusions}

In this study, five 3D re-entrant $(\mathrm{RH})$ structures made of polyamide with varying geometries were manufactured and SHPB impact tests were conducted with different impact velocities. The impact loading process can be explained and understood deeply by analysing the waveform of incident wave, reflection wave and transmitted wave. The test results of stress-strain curves, energy absorption ability and failure mode under impact loading were analysed and compared with the quasi-static test results. Among the tested specimens, both the strength and energy absorption capacity of all impact specimens were significantly improved compared with quasi-static specimens. C2 displayed the strongest energy absorption capacity, which was $1.701 \mathrm{~J} / \mathrm{cm}^{3}$ at the impact velocity of $22 \mathrm{~m} / \mathrm{s}$ and was 5.1 times that in the quasi-static test. The energy absorption of $C 5$ was $1.222 \mathrm{~J} / \mathrm{cm}^{3}$ at the impact velocity of $22 \mathrm{~m} / \mathrm{s}$ and was 15.7 times that in the quasi-static test. C3 displayed the lowest flow stress and the weakest energy absorption capacity due to the half-reduced thickness of the oblique strut cross section $\left(t_{2}\right)$, which was $0.537 \mathrm{~J} / \mathrm{cm}^{3}$ at the impact velocity of $22 \mathrm{~m} / \mathrm{s}$ and was 5.8 times that in the quasi-static test. It was found that the structural geometric parameters had a great effect on the peak yield stress, flow stress and energy absorption capacity of the impact specimen. In general, a smaller re-entrant angle $(\theta)$ can improve the yield stress, peak stress and energy absorption ability, such as C2. A longer horizontal length of the oblique strut $\left(L_{0}\right)$ is helpful to improve the peak stress and energy absorption capacity, such as C5. However, the obvious weakening of structural elements is detrimental to the strength and energy absorption capacity, such as C3. The test 
results can provide a reference for the structural optimization design of similar 3D auxetic structures under medium impact velocity. The trial specimens are limited and more works are required in the future to provide more accurate design predictions.

Author Contributions: Conceptualization, J.C.; methodology, W.T.; software, W.T.; validation, J.C. and S.P.; formal analysis, W.T.; investigation, S.P.; resources, J.C. and W.T.; data curation, W.T.; writing—original draft preparation, J.C. and W.T.; writing—review and editing, J.C.; visualization, W.T.; supervision, J.C.; project administration, J.C.; funding acquisition, J.C. All authors have read and agreed to the published version of the manuscript.

Funding: This work was supported by the Guangdong Province higher vocational college high-level professional group construction project, grant number GSPZYQ2020015.

Data Availability Statement: The raw/processed data required to reproduce these findings cannot be shared at this time as the data also form part of an ongoing study.

Conflicts of Interest: The authors declare no conflict of interest.

\section{References}

1. Liu, Y.P.; Hu, H. A review on auxetic structures and polymeric materials. Sci. Res. Essays 2010, 5, 1052-1063.

2. Evans, K.E.; Alderson, A. Auxetic materials: Functional materials and structures from lateral thinking! Adv. Mater. 2000, 12, 617-628. [CrossRef]

3. Lakes, R. Foam Structures with a Negative Poisson's Ratio. Science 1987, 235, 1038-1040. [CrossRef]

4. Scarpa, F.; Panayiotou, P.; Tomlinson, G. Numerical and experimental uniaxial loading on in-plane auxetic honeycombs. J. Strain Anal. Eng. Des. 2000, 35, 383-388. [CrossRef]

5. Alderson, K.L.; Simkins, V.R.; Coenen, V.L.; Davies, P.J.; Alderson, A.; Evans, K.E. How to make auxetic fibre reinforced composites. Phys. Status Solidi (b) 2005, 242, 509-518. [CrossRef]

6. Choi, J.B.; Lakes, R.S. Fracture toughness of re-entrant foam materials with a negative Poisson's ratio: Experiment and analysis. Int. J. Fract. 1996, 80, 73-83. [CrossRef]

7. Bianchi, M.; Scarpa, F.L.; Smith, C.W. Stiffness and energy dissipation in polyurethane auxetic foams. J. Mater. Sci. 2008, 43, 5851-5860. [CrossRef]

8. Bezazi, A.; Scarpa, F. Mechanical behaviour of conventional and negative Poisson's ratio thermoplastic polyurethane foams under compressive cyclic loading. Int. J. Fatigue 2007, 29, 922-930. [CrossRef]

9. Scarpa, F.; Ciffo, L.G.; Yates, J.R. Dynamic properties of high structural integrity auxetic open cell foam. Smart Mater. Struct. 2004, 13, 49. [CrossRef]

10. Scarpa, F.; Bullough, W.A.; Lumley, P. Trends in acoustic properties of iron particle seeded auxetic polyurethane foam. Proc. Inst. Mech. Eng. Part C J. Mech. Eng. Sci. 2004, 218, 241-244. [CrossRef]

11. Shokri Rad, M.; Prawoto, Y.; Ahmad, Z. Analytical solution and finite element approach to the 3D re-entrant structures of auxetic materials. Mech. Mater. 2014, 74, 76-87. [CrossRef]

12. Li, Y.; Harrysson, O.; West, H.; Cormier, D. Mechanical properties of 3D re-entrant honeycomb auxetic structures realized via additive manufacturing. Int. J. Solids Struct. 2015, 69-70, 475-490.

13. Wang, X.-T.; Wang, B.; Li, X.-W.; Ma, L. Mechanical properties of 3D re-entrant auxetic cellular structures. Int. J. Mech. Sci. 2017, 131-132, 396-407. [CrossRef]

14. Wang, X.-T.; Li, X.-W.; Ma, L. Interlocking assembled 3D auxetic cellular structures. Mater. Des. 2016, 99, 467-476. [CrossRef]

15. Dikshit, V.; Nagalingam, A.P.; Yap, Y.L.; Sing, S.L.; Yeong, W.Y.; Wei, J. Crack monitoring and failure investigation on inkjet printed sandwich structures under quasi-static indentation test. Mater. Des. 2018, 137, 140-151. [CrossRef]

16. Dikshit, V.; Nagalingam, A.P.; Yap, Y.L.; Sing, S.L.; Yeong, W.Y.; Wei, J. Investigation of Quasi-Static Indentation Response of Inkjet Printed Sandwich Structures under Various Indenter Geometries. Materials 2017, 10, 290. [CrossRef]

17. Elipe, Á.; Carlos, J.; Lantada, A.D. Comparative study of auxetic geometries by means of computer-aided design and engineering. Smart Mater. Struct. 2012, 21, 105004. [CrossRef]

18. Wan, H.; Ohtaki, H.; Kotosaka, S.; Hu, G. A study of negative Poisson's ratios in auxetic honeycombs based on a large deflection model. Eur. J. Mech.-A/Solids 2004, 23, 95-106. [CrossRef]

19. Levy, O.; Krylov, S.; Goldfarb, I. Design considerations for negative Poisson ratio structures under large deflection for MEMS applications. Smart Mater. Struct. 2006, 15, 1459. [CrossRef]

20. Masters, I.G.; Evans, K.E. Models for the elastic deformation of honeycombs. Compos. Struct. 1996, 35, 403-422. [CrossRef]

21. Dos Reis, F.; Ganghoffer, J.F. Equivalent mechanical properties of auxetic lattices from discrete homogenization. Comput. Mater. Sci. 2012, 51, 314-321. [CrossRef]

22. Essassi, K.; Rebiere, J.-L.; el Mahi, A.; Souf, M.A.B.; Bouguecha, A.; Haddar, M. Dynamic Characterization of a Bio-Based Sandwich with Auxetic Core: Experimental and Numerical Study. Int. J. Appl. Mech. 2019, 11, 1950016. [CrossRef] 
23. Li, C.; Shen, H.-S.; Wang, H.; Yu, Z. Large amplitude vibration of sandwich plates with functionally graded auxetic 3D lattice core. Int. J. Mech. Sci. 2020, 174, 105472. [CrossRef]

24. Fíla, T.; Zlámal, P.; Jiroušek, O.; Falta, J.; Koudelka, P.; Kytýř, D.; Doktor, T.; Valach, J. Impact Testing of Polymer-filled Auxetics Using Split Hopkinson Pressure Bar. Adv. Eng. Mater. 2017, 19, 1700076. [CrossRef]

25. Ngo, T.D.; Kashani, A.; Imbalzano, G.; Nguyen, K.T.Q.; Hui, D. Additive manufacturing (3D printing): A review of materials, methods, applications and challenges. Compos. Part B Eng. 2018, 143, 172-196. [CrossRef]

26. Ligon, S.C.; Liska, R.; Stampfl, J.; Gurr, M.; Mulhaupt, R. Polymers for 3D Printing and Customized Additive Manufacturing. Chem. Rev. 2017, 117, 10212-10290. [CrossRef] [PubMed]

27. EOS. Available online: https:/ / eos.materialdatacenter.com/eo/en (accessed on 10 August 2020).

28. Chen, J.; Chen, W.; Hao, H.; Huan, S.; Tao, W. Mechanical behaviors of 3D re-entrant honeycomb polyamide structure under compression. Mater. Today Commun. 2020, 24, 101062. [CrossRef]

29. Bacon, C. An experimental method for considering dispersion and attenuation in a viscoelastic Hopkinson bar. Exp. Mech. 1998, 38, 242-249. [CrossRef]

30. Chen, W.W. Experimental Methods for Characterizing Dynamic Response of Soft Materials. J. Dyn. Behav. Mater. 2016, 2, 2-14. [CrossRef]

31. Wang, L.; Labibes, K.; Azari, Z.; Pluvinage, G. Generalization of split Hopkinson bar technique to use viscoelastic bars. Int. J. Impact Eng. 1994, 15, 669-686. [CrossRef]

32. Zhao, H.; Gary, G. A three dimensional analytical solution of the longitudinal wave propagation in an infinite linear viscoelastic cylindrical bar. Application to experimental techniques. J. Mech. Phys. Solids 1995, 43, 1335-1348. [CrossRef]

33. Wang, L.; Lai, H.; Wang, Z.; Yang, L. Studies on nonlinear visco-elastic spherical waves by characteristics analyses and its application. Int. J. Impact Eng. 2013, 55, 1-10. [CrossRef]

34. Butt, H.S.U.; Xue, P. Determination of the wave propagation coefficient of viscoelastic SHPB: Significance for characterization of cellular materials. Int. J. Impact Eng. 2014, 74, 83-91. [CrossRef]

35. Chen, J.; Tao, W.; Huan, S. An improved generalized Lagrangian analysis method for attenuating waves. AIP Adv. 2019, 9, 085214. [CrossRef] 